\title{
L'interruption et le chevauchement dans le débat politique télévisé de l'entre-deux-tours de l'élection présidentielle française de 2017.
}

\author{
Par \\ Mohamed Ahmed Sayed HAMZA* \\ dr.mohamedhamza.fr@alsun.luxor.edu.eg
}

\section{Résumé}

Cet article a pour objectif de retracer les abus commis par les participants lors du débat politique télévisé de l'entre-deux-tours de l'élection présidentielle française de 2017 et le dysfonctionnement interactionnel qui en résulte dans le système des tours de parole. Il se penche particulièrement sur la manière selon laquelle les participants interviennent et s'emparent de la parole, il s'agit donc de l'analyse des interruptions et des chevauchements de parole dans ce débat présidentiel. Pour y arriver, nous jetons tout d'abord la lumière sur le système des tours de parole, le principe d'alternance des tours et les règles qui le suivent pour garantir le fonctionnement idéal de l'interaction. Ensuite, nous focalisons l'attention sur les interruptions et les chevauchements de parole dont les fins sont diverses. Nous essayons alors, à travers l'analyse de différents extraits du débat, de montrer pour quelles raisons ces comportements conversationnels sont utilisés par les différents participants et quels rôles ils peuvent jouer dans un débat politique télévisé décrit comme le plus violent et le plus brutal dans l'histoire des débats politiques en France.

${ }^{*}$ Université de Louxor, Egypte.

(*) L'analyse conversationnelle est un courant qui naît en Californie dans les années (L'interruption et le chevauchement dans le débat...) Mohamed HAMZA 


\section{Avertissement}

Le présent article vise à mettre en lumière les ratés du système des tours à travers l'étude des phénomènes discursifs - l'interruption et le chevauchement - dans le débat politique télévisé 2017 entre E. Macron et M. Le Pen. Vu le manque des références et des écrits français concernant ce domaine, nous avons dû parfois consulter des références anglaises. Donc, les citations anglaises ont été traduites en français par l'auteur de cet article. Ces traductions ont ensuite été numérotées tout au long de cet article, tandis que les citations originales ont été incluses en annexe à la fỉn de l'article.

L'interruption et le chevauchement sont beaucoup représentés lors de ce débat présidentiel. Afin de les analyser, nous choisissons les extraits les plus remarquables qui mettent fortement en évidence ces phénomènes. La numérotation des prises de parole est, dans les exemples à traiter, indispensable pour que l'analyse soit claire et structurée et ne dérange donc pas le lecteur.

\section{- Convention de transcription}

Compte tenu de ce type d'interaction verbale et la nature de l'étude qui s'applique à celle-ci, nous devons suivre un système de convention de transcription qui comprend des symboles mieux adaptés à la représentation des interruptions, des chevauchements, des pauses etc. Ce système de convention de transcription sera respecté jusqu'à la fìn du

(L'interruption et le chevauchement dans le débat...) Mohamed HAMZA 
travail. Voyons alors les symboles utilisés dans l'analyse de notre corpus:

\begin{tabular}{|l|l|}
\hline$/ /$ & Interruption sans chevauchement. \\
\hline // [soulignement] & Interruption avec chevauchement. \\
\hline [soulignement] & Discours chevauchant. \\
\hline$\rightarrow$ & L'interrupteur. \\
\hline+++ & Pause très brève, pause brève. \\
\hline $\mathbf{X} \mathbf{X X ~ X X X}$ & Mot inaudible, deux mots, trois mots.... \\
\hline$\approx \approx$ & Allongement syllabique. \\
\hline
\end{tabular}




\section{Introduction}

Dans leur article fondateur de l'analyse conversationnelle $^{(*)}$, H. Sacks, E. Schegloff et G. Jefferson (SACKS, JEFFERSON, \& SCHEGLOFF, 1974, pp. 700701) ont développé leur modèle de l'alternance des tours de parole permettant de comprendre, au-delà de la perturbation apparente de la parole en interaction, les principes systémiques qui régissent son organisation. $\mathrm{Ce}$ modèle "s'articule autour de la construction des tours où la prise de parole les fait se succéder. Dans cette hypothèse, le tour de parole devient l'unité fondamentale de l'analyse conversationnelle.» (PLETY, 1993, p. 99) A partir de cet article, C. Kerbrat-Orecchioni (KERBRAT-ORECCHIONI, 1990, pp. 159-182) présente et discute le principe d'alternance dans son fonctionnement, ses réglages ainsi que ses ratés dans le cas de la violation de l'une de ses règles qui garantissent le fonctionnement idéal de l'interaction.

L'objectif de cet article est d'aborder l'interruption et le chevauchement comme des ratés du système des tours de parole dans le genre du discours «débat politique télévisé». Il s'agit done d'étudier deux phénomènes langagiers résultant de

${ }^{(*)}$ L'analyse conversationnelle est un courant qui naît en Californie dans les années 1960 grâce au travail de Sacks, Schegloff, Jefferson et d'autres, dans un contexte de collaboration avec Garfinkel, fondateur de l'ethnométhodologie. Ce qui le caractérise est une attention pour l'organisation du langage et de l'action au sein de l'interaction sociale, tels qu'ils s'y organisent de manière localement située. (MONDADA, 2017, p. 182)

(L'interruption et le chevauchement dans le débat... ) Mohamed HAMZA 
la transgression de l'une des règles du principe d'alternance des tours, et plus précisément la transgression des deux règles selon lesquelles «La fonction locutrice doit être occupée successivement par diffèrents acteurs.» et "Une seule personne parle à la fois». Le corpus choisi est le débat politique télévisé de l'entre-deux-tours de l'élection présidentielle française de $2017^{(*)}$. Le choix du corpus est motivé par le fait que ce débat ultra-tendu est spécialement un champ fertile de tels dysfonctionnements interactionnels, à tel point qu'il est décrit comme le plus violent, rugueux et désordonné dans l'histoire de la France. C'est ce qui conduit Adam Nossiter à le décrire comme «un cas de violent combat verbal: les deux fïnalistes se parlaient avec colère, s'interrompaient, remuaient les poings, pointaient du doigt, abandonnant les modérateurs confus et impuissants. (Notre traduction)(1)» (NOSSITER, 2017)

\section{Contextualisation du débat en question}

Le corpus choisi appartient au genre du discours «débat politique télévisé». Celui-ci présente «un

${ }^{(*)}$ Ce débat est disponible sur la plateforme (YouTube) sur les liens:

- https://www.youtube.com/watch?v=i5aqL7FBxyI, consulté du $(1 / 12 / 2019)$ jusqu'au $(15 / 6 / 2020)$

- https://www.youtube.com/watch?v=iOAbBdlWgz0, consulté du $(1 / 12 / 2019)$ jusqu'au $(15 / 6 / 2020)$

Le verbatim est encore disponible en ligne sur le lien:

- https://www.vie-publique.fr/discours/203174-debat-televise-entre-m-emmanuelmacron-et-mme-marine-le-pen-candidats, consulté du (1/12/2019) jusqu'au $(15 / 6 / 2020)$

(L'interruption et le chevauchement dans le débat... ) Mohamed HAMZA 
emboîtement énonciatif entre une interaction en contact l'échange entre des personnalités politiques et un/des animateur(s) - et une scène médiatisée, construite entre les participants au débat et les téléspectateurs-électeurs.» (SANDRÉ, 2011, p. 13) Il s'agit d'un face-à-face télévisé, dont le premier s'est organisé en 1947, entre les deux finalistes à la présidentielle française. On est alors devant un événement politique et médiatique hyper important attendu par les électeurs et suivi par les téléspectateurs. L'importance tient au fait que de tels débats permettent aux téléspectateursélecteurs de bien connaître de près les projets des candidats et le mécanisme de la mise en œuvre.

S'agissant des paramètres de cette situation communicative, il est à remarquer que le débat se focalise essentiellement sur des participants, un cadre spatio-temporel et un but. Par rapport aux participants, le débat s'articule principalement autour des deux finalistes E. Macron (le candidat d'En Marche) et M. Le Pen (la candidate du Front National) d'une part, et un duo de modérateurs Nathalie Saint-Cricq (France 2) et Christophe Jakubyszyn (TF1) d'autre part. À cela s'ajoutent les téléspectateurs-électeurs français qui forment le récepteur direct. Le débat a eu lieu sur le plateau du (studio de La Plaine-Saint-Denis) le 3/5/2017 à $21 \mathrm{~h}$.. Le but principal du débat est la confrontation entre deux visions appartenant aux candidats débatteurs en vue de 
présenter leurs projets électoraux. Tant bien que mal, chaque candidat essaie de convaincre les indécis et marquer les esprits afin de gagner un grand nombre d'électeurs.

\section{Problématique et méthodologie}

L'interruption et le chevauchement de parole représentent, dans ce débat présidentiel, une problématique très importante dans la mesure où ces phénomènes langagiers entraînent des dysfonctionnements interactionnels ou des ratés du système des tours. Plusieurs questions sont lors à poser: Quels sont les types les plus fréquents des chevauchements et des interruptions et comment se manifestent-ils dans ce débat? Quelles sont les raisons qui poussent les participants à effectuer ces dysfonctionnements interactionnels? Pourquoi ces phénomènes prennent-ils une forme particulière dans ce débat? Est-ce que les modérateurs ont le droit d'interrompre ou de se chevaucher avec le locuteur en place et pourquoi? Les modérateurs jouent-ils un rôle dans la réduction de ces phénomènes? Ces chevauchements et ces interruptions influencent-ils le bon fonctionnement du débat?

Le présent article se situe dans le cadre de l'analyse du discours et se sert de principaux outils descriptifs de l'analyse conversationnelle en reposant sur les propositions de la linguistique interactionnelle. La démarche adoptée est alors qualitative dans la mesure où nous visons à décrire et

(L'interruption et le chevauchement dans le débat... ) Mohamed HAMZA 
interpréter ces comportements interactionnels et cela à travers l'analyse de la teneur du discours qui accompagne ces phénomènes langagiers. Pour mener à bien ce travail, plusieurs points à traiter: le système des tours de parole, le principe d'alternance des tours et les ratés du système des tours de parole qui comprennent l'analyse des interruptions et des chevauchements de parole.

\section{Système des tours de parole}

L'interaction verbale, en l'occurrence le débat politique télévisé, suppose une situation de communication orale, le plus souvent en face à face, dans laquelle deux ou plusieurs participants échangent des propos à tour de rôle. Chaque participant a un statut et un rôle au cours de l'interaction. Cette interaction verbale est considérée comme des conduites rangées et se déroule conformément aux règles de procédure. Celles-ci qui régissent les interactions verbales sont, selon $\mathrm{C}$. Kerbrat-Orecchioni (KERBRAT-ORECCHIONI, 1996, p. 28 ), de nature très diverse dont on distingue trois grandes catégories:

- Règles qui permettent la gestion de l'alternance des tours de parole.

- Règles qui régissent l'organisation structurale de l'interaction.

- Règles enfin qui interviennent au niveau de la relation interpersonnelle.

(L'interruption et le chevauchement dans le débat...) Mohamed HAMZA 
D'après cette linguiste (KERBRAT-ORECCHIONI, 1996, p. 26), le système de tours de parole est vu comme «un système de droits et de devoirs» ou un «un système d'attente.» Cela signifie que le système de prise de tour suppose que chaque locuteur a le droit de conserver son rôle de parole pendant un certain temps, et il doit également laisser la parole à un certain moment pour l'autre locuteur afin de prendre son droit de parole. Ce système permet donc aux tours de parole de s'enchaîner et contribue à réduire les silences, les chevauchements et les interruptions. Dans la conversation ordinaire, cet arrangement n'est pas déterminé à l'avance, mais il est organisé au moment de construire les échanges et selon le mécanisme d'alternance des tours de parole.

Néanmoins, dans le genre débat politique télévisé, la taille et l'ordre des tours sont prédéterminés par les modérateurs qui expliquent clairement, dès le début, les règles du débat et le fonctionnement des tours de parole. En tant que modérateurs du débat, ils prennent en charge plusieurs tâches, y compris principalement la distribution de la parole et le contrôle des tours de parole. Grâce au contrôle des tours, «les modérateurs donnent à leurs invités la possibilité de prendre la parole alternativement et de bénéficier d'un nombre égal de tours.» (MARINESCU, 2017, p. 35) D'autre part, le manque de contrôle des tours par les

(L'interruption et le chevauchement dans le débat...) Mohamed HAMZA 
modérateurs, particulièrement dans les débats présidentiels, pourrait provoquer le chaos sur le plateau. Pour cette raison, seuls les modérateurs les plus expérimentés qui y sont invités à réaliser.

Puisque toute interaction verbale se présente comme une succession des tours de parole, nous allons aborder le principe d'alternance des tours dans la mesure où le corps même de l'interaction doit suivre ce principe qui règle les tours de parole. Nous allons aussi voir si ce principe est facile à appliquer à de tels débats et si les participants respectent ce principe ou ils le transgressent.

\section{Principe d'alternance des tours de parole}

Sacks, Schegloff et Jefferson sont parmi les pionniers qui se sont intéressés à l'analyse conversationnelle. Ils ont montré que la conversation doit obéir à certaines règles de base qui servent à l'organisation de la distribution des tours et à la transition harmonieuse d'un tour à l'autre. A ce titre, ils ont présenté, dans leur article célèbre paru en 1974 (SACKS, JEFFERSON, \& SCHEGLOFF, 1974), un modèle d'alternance des tours dans lequel il y a 14 propriétés propres à tout échange de parole. Ce modèle supporte le principe d'alternance des tours qui forme un mécanisme clé pour le bon fonctionnement de l'interaction. Conformément à ce mécanisme, chaque participant prend la parole à son tour pendant un temps précis, alors que l'autre interlocuteur doit

(L'interruption et le chevauchement dans le débat... ) Mohamed HAMZA 
attendre jusqu'à ce qu'il termine son discours pour ensuite prendre son tour de parole et ainsi de suite. C'est ce qui amène $\mathrm{C}$. Kerbrat-Orecchioni à dire que le terme "tours de parole" désigne «le mécanisme d'alternance des prises de parole, [...], la contribution verbale d'un locuteur déterminé à un moment déterminé du déroulement de l'interaction.» (KERBRAT-ORECCHIONI, 1990, p. 160)

Le principe d'alternance est alors régi par un certain nombre de règles qui se chevauchent et qui doivent être respectées simultanément afìn de mener l'interaction correctement. Le respect de ces règles assure la fluidité des échanges et permet à la parole d'être partagée par les différents participants de manière successive et incessante. Christine Béal (BEAL, 2010, p. 86) montre, de sa part, que la distribution de la parole entre les participants se réalise selon un certain nombre de principes qui relèvent de la coopération entre les locuteurs et qui assurent une alternance des tours de parole: principe de l'équilibre entre les locuteurs, principe de "chacun son tour" et principe d'un intervalle minimum entre les tours.

D'autre part, la sélection du tour suivant «se fait à des points d'achèvement potentiel qui sont des places transitionnelles comme des silences ou la fin d'une question.» (GREVISSE \& Da SILVA-GENEST, 2018, p. 385) Donc, le changement de tour de parole «doit normalement s'effectuer 
à un point de transition possible.» (KERBRATORECCHIONI, 1996, p. 30) Ce point de transition doit être inscrit dans l'énoncé par l'un des signaux de fin de tour. Mais, ces derniers "sont variés, de nature différente (lexicaux, syntaxiques, pragmatiques, prosodiques, mimo-gestuels) et fonctionnent en général de façon combinée et cumulative.» (BEAL, 2010, p. 94) Tous ces signaux sont produits par le locuteur en place.

En regardant la rediffusion de ce débat, nous trouvons que les tours sont allouées, selon les règles médiatiques régissant le débat, par l'un de deux modérateurs qui «occupe en quelques sorte la fonction de distributeur officiel des tours.» (KERBRAT-ORECCHIONI, La conversation, 1996, p. 30) Soulignons aussi que l'alternance des tours nous semble très difficile à appliquer dans un débat présidentiel inédit dans l'histoire de la France. Ce débat ultra-tendu est décrit comme le plus rugueux et désordonné parmi les autres débats précédents en France. C'est peut-être dû au fait que ce débat est entre deux visions tout à fait contradictoires, et chaque candidat adopte un projet opposé à celui de l'autre. Macron est libéral, pro-européen et conforme à la mondialisation. Alors que Le Pen est un isolationniste, antiimmigration et anti-européen. Par conséquent, le débat se transforme en guerre verbale et les tours de parole sont à la fois plus embrouillés et plus irréguliers. L'exemple typique, 
par rapport au principe d'alternance des tours et les règles qui l'organisent, n'a eu lieu qu'entre les modérateurs à l'ouverture du débat:

\begin{tabular}{|l|l|}
\hline (1) & $\begin{array}{l}\text { M. Jakubyszyn: Bonsoir Nathalie et bonsoir à } \\
\text { tous. (...) Pendant deux heures, nous évoquerons } \\
\text { l'économie, le terrorisme, l'éducation et l'Europe. } \\
\text { (...), disposer d'une carte blanche pour évoquer } \\
\text { le thème de votre choix + } \\
\mathbf{M}^{\text {me }} \text { Saint-Cricq: Alors notre rôle sera de } \\
\text { veiller sur la stricte égalité de votre temps de } \\
\text { parole et également à l'équité de vos échanges + } \\
\text { (2) Jakubyszyn: Vous pouvez également suivre } \\
\text { ce débat sous-titré sur TF1, France 2, LCI et } \\
\text { France info. Et en langue des signes sur l'antenne } \\
\text { de France info et le site internet de LCI + } \\
\text { Me Saint-Cricq: Alors le tirage au sort vous a } \\
\text { désigné Madame Marine Le Pen pour } \\
\text { commencer. C'est donc vous, Monsieur } \\
\text { Emmanuel Macron, qui conclurez + } \\
\text { M. Jakubyszyn: Mais avant de nous intéresser à } \\
\text { vos propositions, une première question: (...) } \\
\text { quel est votre état d'esprit? C'est donc à vous, } \\
\text { Marine Le Pen + }\end{array}$ \\
\hline
\end{tabular}

Cet exemple montre la succession idéale des tours de parole, puisque les deux modérateurs les accaparent à tour de 
rôle. Il comprend quatre tours de parole juxtaposés sans interruption ni chevauchement ni silence prolongé entre les tours. C'est parce que ces tours de parole sont organisés et prédéterminés par les modérateurs et s'effectuent de façon automatique. Remarquons comment M. Jakubyszyn prend son tour de parole à un moment donné, alors que $\mathrm{M}^{\mathrm{me}}$ SaintCricq l'attend jusqu'à la fin de son tour. Puis cette dernière commence à parler dès que $\mathrm{M}$. Jakubyszyn a terminé son tour et ainsi de suite. Ce qui capte notre attention, c'est que le changement de tour entre les deux s'effectue seulement par des signaux de fin de tour de nature verbale (la complétude syntactico-sémantique des énoncés) et prosodique (la courbe intonative puis la chute de la voix). Tandis que les signaux non-verbaux sont totalement absents, puisque le modérateur en place regarde directement vers l'écran en parlant et l'autre modérateur attend la chute de la voix pour commencer. Passons aussi à autre exemple où les participants respectent uniquement le système des tours à l'ouverture du débat: 


\begin{tabular}{|c|c|}
\hline (1) & $\begin{array}{l}\text { M. Jakubyszyn: Mais avant de nous intéresser à } \\
\text { vos propositions, une première question: (...) } \\
\text { quel est votre état d'esprit? C'est donc à vous, } \\
\text { Marine Le Pen + }\end{array}$ \\
\hline (2) & $\begin{array}{l}\text { M }^{\text {me }} \text { Le Pen: (...) Monsieur Macron est le } \\
\text { candidat de la mondialisation sauvage, de } \\
\text { l'upérisation, de la précarité, de la brutalité } \\
\text { sociale, de la guerre de tous contre tous, (...). Je } \\
\text { pense que cette période, en réalité, de } \\
\text { clarification a été profondément utile aux } \\
\text { Français pour faire un choix }+\end{array}$ \\
\hline (3) & $\begin{array}{l}\text { M. Jakubyszyn: C'est donc à vous, Emmanuel } \\
\text { Macron, de me donner votre état d'esprit à } \\
\text { quelques jours du scrutin + }\end{array}$ \\
\hline$(5) \rightarrow$ & $\begin{array}{l}\text { M. Macron: Ecoutez, vous avez donc démontré } \\
\text { que vous n'êtes en tout cas pas la candidate de } \\
\text { l'esprit de finesse, (...). Ce sont ceux-là que je } \\
\text { veux conduire, et c'est l'incapacité des } \\
\text { gouvernements depuis tant et tant d'années }+/ / \\
\mathbf{M}^{\text {me }} \text { Le Pen: dans le vôtre }\end{array}$ \\
\hline$(7) \rightarrow$ & $\begin{array}{l}\text { M. Macron: Depuis } 30 \text { ans, je n'ai jamais été ni } \\
\text { Premier ministre, ni président de la République // } \\
\mathbf{M}^{\mathbf{m e}} \text { Le Pen: ministre }\end{array}$ \\
\hline (8) & $\begin{array}{l}\text { M. Macron: (...) C'est cela la France qui nous } \\
\text { ressemble, pas la vôtre }+\end{array}$ \\
\hline
\end{tabular}

(L'interruption et le chevauchement dans le débat...) Mohamed HAMZA 
Cet exemple est moins typique par rapport aux règles, déjà mentionnées, qui assurent l'alternance des tours. C'est parce que ces règles sont seulement respectées en (1), (2), (3) et (4). Mais en (5) et (7), il y a un dysfonctionnement interactionnel, étant donné que M. Le Pen coupe délibérément la parole à Macron 2 fois successives sans chevauchement. Concernant la répartition des tours de parole, elle est gérée par $M$. Jakubyszyn, l'un de deux modérateurs de ce débat. Celui-ci a premièrement donné la parole à Marine Le Pen qui a gardé la parole un certain temps; mais aussi elle la lui a rendue une autre fois à moment donné. D'autre part, Macron l'a laissée parler jusqu'à la fin sans aucune interruption. Puis, M. Jakubyszyn a donné la parole à Macron qui a pris son tour de parole, mais M. Le Pen a transgressé le système des tours en l'interrompant. Cet extrait montre comment le débat s'est ouvert sur des échanges très tendus entre les deux candidats débateurs, c'est ce qui reflète la stratégie de guerre totale que chaque candidat emploie et qui se concentre sur le bombardement de l'adversaire sans relâche.

Par rapport au changement de tour de parole d'un participant à l'autre, il est à remarquer que M. Jakubyszyn, M. Le Pen et E. Macron ont fini tous les deux le tour de parole par des signaux de nature verbale sous la forme des énoncés: (C'est donc à vous, (...) / Je pense que cette

(L'interruption et le chevauchement dans le débat...) Mohamed HAMZA 
période, (...). / C'est cela la France qui nous ressemble, pas la vôtre). Ces énoncés-ci s'accompagnent de codes mimogestuels qui sous-tendent les mimiques faciales et les gestes (le regard, le sourire, le changement de posture et le mouvement des mains). Le regard ou le contact oculaire, avec les autres participants, est l'un des signes non-verbaux auquel a recouru le modérateur pour distribuer la parole et aussi les candidats pour exprimer la fin du tour. Ces énoncés sont aussi suivis par des signaux de nature phonétiques, surtout prosodiques qui commencent par la courbe intonative et se terminent par la chute de la voix. Tous ces signaux sont fonctionnés de manière ordonnée et complémentaire.

Après avoir examiné notre corpus, il est à remarquer que le principe d'alternance des tours est uniquement respecté au début du débat soit par les deux finalistes, soit par les modérateurs. Ceux-ci recourent, pour de nombreuses raisons, à la violation de l'une des règles constituant ce principe soit par le chevauchement ou l'interruption. C'est ce que l'on appelle les ratés du système des tours ou les dysfonctionnements interactionnels où les participants parlent simultanément avec leurs partenaires ou les interrompent. Tel sera le point d'analyse subséquent.

(L'interruption et le chevauchement dans le débat...) Mohamed HAMZA 


\section{Ratés du système des tours de parole}

C'est la notion que C. Kerbrat-Orecchioni (KERBRAT-ORECCHIONI, 1990, p. 172) a donnée aux dysfonctionnements interactionnels résultant de la violation d'une règle du bon fonctionnement de l'interaction. Ces ratés renvoient done, dans une interaction, à «la transgression des règles interactionnelles. Ces règles garantissent le bon fonctionnement d'une interaction et concernent notamment la succession des prises de parole et l'organisation structurale des interventions.» (DANLER, ILIESCU, \& SILLER, 2010, p. 251) Il s'agit donc des phénomènes discursifs transgressant les règles et les principes qui garantissent le bon fonctionnement de l'interaction, surtout le principe d'alternance des tours de parole.

Les quatorze règles du principe d'alternance des tours, proposées par Sacks et ses collègues (SACKS, JEFFERSON, \& SCHEGLOFF, 1974, pp. 700-701), sont récapitulées par C. Kerbrat-Orecchioni (KERBRAT-ORECCHIONI, 1996, pp. 28-29) en trois règles. Chacune de ces règles se rapporte à un dysfonctionnement interactionnel qui a lieu dans le cas de la violation délibérée de ces règles comme le montre le tableau ultérieur:

(L'interruption et le chevauchement dans le débat...) Mohamed HAMZA 


\begin{tabular}{|l|l|}
\hline \multicolumn{1}{|c|}{ La règle } & $\begin{array}{l}\text { Phénomène produit de la } \\
\text { transgression de la règle }\end{array}$ \\
\hline $\begin{array}{l}\text { - La fonction locutrice doit } \\
\text { être occupée successivement } \\
\text { par différents acteurs. }\end{array}$ & • L'interruption \\
\hline $\begin{array}{l}\text { - Une seule personne parle à } \\
\text { la fois. }\end{array}$ & $\begin{array}{l}\text { - Le chevauchement de } \\
\text { parole }\end{array}$ \\
\hline $\begin{array}{l}\text { - Il y a toujours une personne } \\
\text { qui parle. }\end{array}$ & $\begin{array}{l}\text { Le silence prolongé entre } \\
\text { deux tours (ou gap) }\end{array}$ \\
\hline
\end{tabular}

Ce qu'il est à remarquer dans ce tableau, c'est la présence de 3 règles qui résument le principe d'alternance des tours de parole. La transgression de l'une de ces règles entraîne automatiquement un dysfonctionnement interactionnel ou un raté du système des tours. Ce que nous allons traiter principalement dans ce point de recherche, ce sont les deux premiers phénomènes. C'est en effet parce que le silence prolongé entre deux tours (ou gap) n'est pas si fortement présent dans tel genre du discours. D'autre part, l'interruption et le chevauchement sont deux stratégies interactionnelles ayant une forte présence, très particulièrement dans ce débat présidentiel constituant notre corpus. Nous allons donc maintenant envisager le chevauchement de parole.

(L'interruption et le chevauchement dans le débat...) Mohamed HAMZA 


\subsection{Chevauchement de parole}

En règle générale, toute interaction doit suivre une alternance des tours de parole, et chaque locuteur parle à son tour. Pourtant, il est possible que plusieurs personnes parlent simultanément. Ces cas sont inévitables et fréquents dans tout échange verbal entre deux ou plusieurs personnes. On parle alors d'un chevauchement de parole. Celui-ci se produit lorsque « $\mathrm{L}_{2}$ se met à parler alors que $\mathrm{L}_{1}$ continue de parler.» (KERBRAT-ORECCHIONI, 1996, p. 32) Donc, il est vu comme une superposition anormale de parole où deux personnes ou plus parlent en même temps. Il s'agit alors d'«un cas de panne conversationnelle allant à l'encontre d'une des règles conversationnelles à savoir que les locuteurs prennent la parole successivement.» (GREVISSE \& Da SILVA-GENEST, 2018, p. 385) Dans ce cas, on trouve que les deux interlocuteurs "semblent s'efforcer de prendre la parole et selon qui est le plus fort, un interlocuteur parvient finalement à obtenir le tour. (Notre traduction) (2)» (PSCHAID, 1993, p. 58)

Dans les paragraphes suivants, nous abordons les différents types d'apparition de chevauchements pour explorer comment les participants à ce débat finissent par entrer en collision les uns avec les autres et parler simultanément lorsqu' "un locuteur parle à la fois" est la règle conversationnelle. En examinant ce phénomène

(L'interruption et le chevauchement dans le débat...) Mohamed HAMZA 
langagier dans le corpus, nous trouvons qu'il est fortement fréquent et que les fins sont très diverses: fin polémique (beaucoup plus représentée dans le corpus), fin coopérative (peu représentée) ou fin concernant la gestion du débat (effectuée seulement par les modérateurs).

Ce phénomène apparaît, d'après $\mathrm{J}$. Sidnell et $\mathrm{T}$. Stivers (SIDNELL \& STIVERS, 2013, pp. 176-177), sous trois formes d'un chevauchement selon la place qu'il occupe pendant le tour du locuteur en cours: (Turn-initial) au début du tour, (Mid-turn) au milieu du tour et (Turn-terminal) à la fin du tour. Pour analyser ce dysfonctionnement interactionnel, il faut tenir compte de la teneur du discours chevauchant et des indices spécifiques (verbaux, prosodiques, mimo-gestuels) qui les accompagnent.

Par rapport au chevauchement (Turn-initial), il se produit au début du tour en cours entre deux locuteurs. Selon J. Sidnell et T. Stivers, «les débuts des tours sont aussi un lieu commun de chevauchement de parole. (Notre traduction)(3)» (SIDNELL \& STIVERS, 2013, pp. 176-177) Ce type de chevauchement apparaît de temps en temps, dans le débat politique de l'entre-deux-tours, entre E. Macron ou M. Le Pen avec l'un des modérateurs. Voilà un exemple où le chevauchement ne se produit pas entre le locuteur en cours (Macron) et son successeur potentiel (M. Le Pen), mais au

(L'interruption et le chevauchement dans le débat...) Mohamed HAMZA 
début entre la dernière et $\mathrm{M}^{\text {me }}$ Saint-Cricq, puis les trois personnes commencent à parler en même temps:

\begin{tabular}{|c|c|}
\hline (1) & $\begin{array}{l}\text { E. Macron: (...) Soit augmenter la dette et à ce } \\
\text { moment-là ce seront nos enfants qui payeront. Je ne } \\
\text { veux aucune de ces solutions, aucune. C'est tout + } \\
\text { M. Le Pen: Vous, vous en avez fait //[trois cents } \\
\text { milliards de dettes pendant votre quinquennat.] }\end{array}$ \\
\hline$(3) \rightarrow$ & $\begin{array}{l}\mathbf{M}^{\text {me }} \text { Saint Cricq: [Alors on va revenir tout à } \\
\text { l'heure. on XX tout à l'heure. On reviendra X] }\end{array}$ \\
\hline (4) & E. Macron: Ça je ne peux, ce n'est pas le cas. \\
\hline (5) & M. Le Pen: [C'est difficile d'assumer son bilan?] \\
\hline (6) & $\begin{array}{l}\text { E. Macron: Moi j'assume totalement mon bilan, le } \\
\text { problème c'est que vous n'//[êtes pas capable de me } \\
\text { citer un cas industriel probant sur mon bilan. Mais }\end{array}$ \\
\hline$(7) \rightarrow$ & $\begin{array}{l}\text { on peut y venir.] } \\
\mathbf{M}^{\text {me }} \text { Saint Cricq: }[\mathrm{Ma}++ \text { M. Le Pen! M. Le Pen! }\end{array}$ \\
\hline & $\begin{array}{l}++ \text { On y reviendra tout à l'heure. E. Macron! Non } \\
\text { mais on ne va pas tourner en boucle.l }\end{array}$ \\
\hline
\end{tabular}

Dans cet exemple, nous remarquons que le début de chevauchement est: (2, 3 et 4). M. Le Pen commence son tour de parole comme successeur potentiel après une pause intra-tour, mais $\mathbf{M}^{\text {me }}$ Saint-Cricq s'entremêle avec elle en parlant pour terminer le discours dans ce domaine, puis Macron répond à $\mathrm{M}$. Le Pen en parlant du fardeau de la dette des dernières années. Le chevauchement commence à 
nouveau et s'étend aux ( 5,6 et 7$)$, puisque les deux candidats débatteurs ainsi que la modératrice parlent simultanément. Les deux finalistes discutent autour des problèmes de l'économie et de la baisse d'impôts selon leur programme électoral, et chacun essaie de réfuter les idées de l'autre. Mais Macron, en tant qu'ancien ministre de l'Économie sous François Hollande, s'exprime sur un ton très confiant accompagné d'un sourire affirmant cette confiance. À cause de cette controverse mutuelle, la modératrice intervient une autre fois pour mettre fin à la discussion à ce stade et passer à un autre thème. Mais, le ton de la modératrice est faible et hésitant, ce qui ne lui permet pas de contrôler la situation.

D'autre part, le chevauchement (Mid-turn) a lieu au milieu du tour de parole où le locuteur suivant transperce le tour en cours et commence à parler alors que le locuteur actuel n'a pas encore fini de parler. Cependant, de tels chevauchements «affiliés au milieu du tour ne sont pas le produit des erreurs concernant la prise de parole. Leur apparition semble plutôt montrer les orientations des intervenants suivants à l'égard de préoccupations comme le contenu du tour actuel, l'adéquation et la progressivité. (Notre traduction)(4)» (SIDNELL \& STIVERS, 2013, p. 177) Dans ce débat, ce type de chevauchement est le plus représenté parmi les deux autres, puisque les deux candidats l'ont souvent utilisé soit pour réagir et réfuter les idées du 
candidat, soit pour justifier sa position sur le thème en discussion. Prenons cet exemple où $\mathrm{M}$. Macron se trouve en chevauchement avec $\mathbf{M}^{\mathrm{me}}$ Le Pen:

\begin{tabular}{|c|c|}
\hline 1) & $\begin{array}{l}\text { E. Macron: (...) Moi j'étais avec les représentants } \\
\text { des salariés, parce que je les respecte. (....) Ils ont } \\
\text { bien compris que vous ne leur proposerez rien }+ \\
\text { M. Le Pen Les salariés? + + // }\end{array}$ \\
\hline- & $\begin{array}{l}\text { E. Macron: [Ils ont bien compris que vous ne leur } \\
\text { proposerez rien] }\end{array}$ \\
\hline ) & $\begin{array}{l}\text { M. Le Pen: [ils vous ont } \approx \text { hué } \approx \text { M. Macron }+, \approx \\
\text { hué } \approx+\text { et ils vous ont dit } \approx \text { dehors } \approx]\end{array}$ \\
\hline 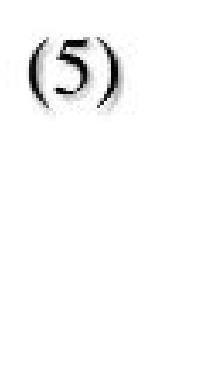 & $\begin{array}{l}\text { E. Macron: [Le soir même, mais oui parce qu'ils } \\
\text { lui aient l'air, et moi je prends cette part de colère } \\
\text { M. Le Pen.] }\end{array}$ \\
\hline ) & $\begin{array}{l}\text { M. Le Pen: [Parce qu'ils savent que toute la } \\
\text { campagne, moi j'ai parlé d'eux. J'ai mis la lumière.] }\end{array}$ \\
\hline 7) & $\begin{array}{l}\text { E. Macron: [Vous ne leur proposerez rien. Vous } \\
\text { jouez avec leur part de colère Madame Le Pen.] Le }\end{array}$ \\
\hline & $\begin{array}{l}\text { posant quoi? } \\
\text { // }\end{array}$ \\
\hline - & $\begin{array}{l}\text { M. Le Pen: On l'a mis sous protection par // [l'État, } \\
\text { le temps de trouver un repreneur.] }\end{array}$ \\
\hline )- & E. Macron: [mais, c'est à se tenir les côtes],$(\ldots)$ \\
\hline
\end{tabular}

Cet exemple montre comment les énoncés $(3,4,5,6$ et la première partie de 7) et (la dernière partie de 8 et la 
dernière partie de 9) sont synchrones. En (1), M. Macron prend son tour de parole jusqu'à la fin sans aucune interruption ni chevauchement. Cependant, quand $\mathrm{M}^{\text {me }}$ Le Pen a commencé à parler, M. Macron a interagi avec elle, exprimant son objection à ce qu'elle dit à propos du face-àface tendu entre Macron et les salariés de Whirlpool. La discussion s'est étendue jusqu'au premier énoncé de (9). Macron tente de prouver qu'il s'intéresse aux salariés grévistes et défend leurs problèmes tout en accusant $\mathrm{M}^{\mathrm{me}} \mathrm{Le}$ d'avoir rencontré ces travailleurs pour prendre quelques selfies et qu'elle ne leur a rien proposé. L'objectif de tel chevauchement est de se défendre et de contester l'autre, et que chaque candidat estime qu'il vaut mieux résoudre la crise de cette entreprise en grève pour gagner plus de votes électoraux. Le ton accompagné du discours chevauchant est assez ascendant. Mais celui de M. Le Pen, surtout en (4), s'accompagne d'un allongement syllabique dans les mots (hué / dehors) qui expriment, selon la teneur de l'énoncé, le sarcasme de l'adversaire.

Cependant, le chevauchement (Turn-terminal) est celui qui se passe juste avant la fin du tour comme le montre $\mathbf{J}$. Sidnell et T. Stivers: «L'auto-sélection du locuteur suivant qui s'oriente à devenir le «premier démarreur» commence souvent un tour suivant légèrement avant la fin du tour en cours, et se retrouve donc en bref chevauchement avec le 
locuteur actuel. De tels chevauchements finaux sont généralement éphémères car leur début est placé à un point où l'achèvement du tour en cours est imminent. (Notre traduction)(5)» (SIDNELL \& STIVERS, 2013, p. 175) Cela tient au fait que le successeur potentiel anticipe la fin du tour grâce à des signaux de fìn de tour émis par le locuteur en place. Dans ce cas, le successeur potentiel prend son tour de parole, alors que le locuteur en place n'a pas encore terminé son tour. Voici un exemple où $\mathbf{M}^{\text {me }}$ Saint-Cricq prend son tour de parole et présente son collègue pour lancer le dossier de l'Europe à discuter, mais M. Jakubyszyn assume la parole alors qu'elle est en train de parler:

\begin{tabular}{|l|l|}
\hline (1) & $\begin{array}{l}\text { M } \\
\text { Che Saint Cricq: (...) On va parler d'Europe avec } \\
\text { comprendre qu'entre vous deux c'est un sujet } \\
\text { important donc ça mérite d'y passer maintenant, + // } \\
\text { si vous n'êtes d'accord, //[Christophe!] } \\
\text { M. Jakubyszyn: Effectivement + [merci Nathalie]. } \\
(2) \rightarrow\end{array}$ \\
\hline
\end{tabular}

Lors de l'examen du chevauchement de parole dans notre corpus, il est à remarquer que ce dysfonctionnement interactionnel est parfois joint par un phénomène langagier, à savoir la répétition du dernier énoncé du discours chevauchant. Ce phénomène a déjà été étudié en détail par $\mathrm{E}$. Schegloff. Selon ce dernier, «Parfois une brève, parfois une 
plus longue répétition, redire ou recyclage d'une partie de l'énoncé se produit dans un tour conversationnel. (Notre traduction)(6)» (SCHEGLOFF, 1987, p. 71) Cette répétition, qui suit un instant du discours chevauchant, se produit dans des environnements de prise de parole compétitive. C'est ce que Jefferson affirme lorsqu'il dit: "Cette procédure semble avoir une certaine régularité d'occurrence après chevauchement concurrentiel. L'une des parties concurrentes annonce des problèmes et démarre explicitement des procédures de réparation en demandant une répétition de l'énoncé de chevauchement de son concurrent. (Notre traduction)(7)» ( JEFFERSON, 2004, p. 52) Dans ce débat présidentiel, E. Macron et $\mathrm{M}$. Le Pen se sont servis de ce procédé plusieurs fois soit pour défendre leur tour de parole, soit pour insister sur la mise en évidence d'une certaine partie de leurs paroles inaudibles pendant le chevauchement. Tel est le cas dans cet exemple:

\begin{tabular}{|l|l|} 
(1) & $\begin{array}{l}\text { M. Le Pen: }(\ldots) \text { M. Macron veut que les } \\
\text { négociations aient lieu par entreprise } / / \quad \approx \text { C'est } \\
\text { fratricide } \approx+\approx \text { C'est fratricide } \approx]\end{array}$ \\
$(2) \rightarrow$ & $\begin{array}{l}\text { E. Macron: [Dites d'abord ce que vous voulez } \\
\text { clairement.... ne déformez pas!] }\end{array}$ \\
(3) & M. Le Pen: C'est fratricide, $(\ldots)$
\end{tabular}

En (1), M. Le Pen assume son tour de parole, mais E. Macron l'interrompt et se chevauche sans aucun signal de fin 
de tour de la part de M. Le Pen. D'ici, le chevauchement a lieu et les énoncés "C'est fratricide....» et "Dites d'abord ce que vous voulez clairement.... ne déformez pas!) sont simultanés. C'est ce qui a conduit $\mathrm{M}$. Le Pen à répéter l'énoncé "C"est fratricide.... 3 fois successives: 2 fois pendant le chevauchement et la troisième lors du redémarrage du tour de parole à nouveau. Cette répétition est accompagnée de l'intonation montante, l'accentuation syllabique, l'allongement syllabique ainsi que les procédés paralinguistiques $^{(*)}$. Telle répétition d'une partie du tour, après la fin de la parole synchrone, permet à $\mathrm{M}$. Le Pen de recommencer son tour sans pause ni chevauchement par la suite. Dans cet exemple, l'objectif de la répétition est de garder le tour de parole d'une part et d'exprimer le refus de la manière de discussion d'autre part.

Après avoir abordé le chevauchement de parole, dans le débat de l'entre-deux-tours de l'élection présidentielle de 2017, il est très évident que ce phénomène interactionnel domine une bonne partie du débat. Vu la nature de ce débat, un grand nombre des chevauchements sont effectués par les candidats et prennent un caractère compétitif, mais le reste se

${ }^{(*)}$ Les procédés paralinguistiques qui doivent rendre l'énonciation verbale univoque, exacte, et ainsi la compléter à l'instant synchrone, relèvent particulièrement les gestes (mouvements des membres supérieurs et de leurs parties), la mimique (mouvements de la face et des organes se trouvant sur la face, mouvements de la tête tout entière), [...]. (SCHULZOVA, 1990, p. 396)

(L'interruption et le chevauchement dans le débat... ) Mohamed HAMZA 
pratique par les modérateurs en vue de gérer et contrôler le débat. C'est peut-être parce que chaque candidat essaie de se défendre et de critiquer les points de vue de l'adversaire pour persuader une grande partie de l'électorat et ainsi gagner plus de votes électoraux. D'autre part, ce discours simultané «qui, au premier abord, paraîtrait incarner "le désordre caractéristique de l'oral" exhibe en réalité l'attention mutuelle des participants envers la possible complétude d'un énoncé et donc envers des statuts de locuteur suivant ou en cours.» (OLOFF, 2008, p. 71)

Tournons-nous à présent vers un autre phénomène interactionnel qui est fortement représenté dans le corpus et considéré comme l'un des ratés du système des tours, à savoir "l'interruption".

\subsection{Interruptions}

C'est le fait de retarder ou d'interrompre quelqu'un qui est en train de parler par un interrupteur. C'est ce dernier qui ne peut plus attendre jusqu'à ce que son partenaire termine son tour de parole. Il s'agit alors d'«une action de couper la parole à quelqu'un; apostrophe, paroles lancées au milieu d'un discours. $\rangle^{(1)}$ De sa part, C. Kerbrat-Orecchioni montre que: «Interrompre autrui, c'est lui "couper" la parole, c'est donc léser son "territoire" et menacer sa "face".»

(1) Dictionnaire de français Larousse, disponible sur le site: https://www.larousse.fr/ dictionnaires /francais/ interruption/43845. Consulté le 10/3/2020.

(L'interruption et le chevauchement dans le débat...) Mohamed HAMZA 
(KERBRAT-ORECCHIONI, 1996, p. 32) Telle interruption se produit dès lors que le locuteur en place qui assume le discours le perd en faveur de son partenaire. Alors, l'interruption est un dysfonctionnement interactionnel violant l'une des règles du principe d'alternance des tours. Pour cette raison, ce phénomène langagier est fortement attaché à la question de la politesse dans les interactions verbales. (KERBRAT-ORECCHIONI, 1996, p. 50)

L'interruption est parfois considérée au moins comme une impolitesse, c'est-à-dire une faute sociale et de communication. C'est peut-être parce que l'autre participant s'empare de la parole et se chevauche le plus souvent avec le locuteur en cours violant le principe d'alternance des tours qui garantit la succession idéale de différents tours de parole. Néanmoins, l'interruption peut aussi être légitime, il s'agit d'une technique de communication, particulièrement en pédagogie. Dans ce cas, elle permet de «provoquer la pensée, de troquer la bêtise, de produire le raisonnement. L'interrupteur «allume», «éclaire», si l'on peut dire, [...], faire «disjoncter» la scène communicationnelle.» (GUEDJ, 2008, p. 26)

L'interruption peut apparaître sous deux types: interruption nette et interruption avec chevauchement. Ça dépend alors du cas dans lequel elle est accompagnée d'un chevauchement ou non. L'interruption nette se produit quand 
l'interrupteur arrive à se glisser au cours d'une pause à l'intérieur d'un tour de la part du locuteur actuel, et par conséquent ce dernier s'arrête de parler. Mais l'interruption avec chevauchement, c'est le cas où l'interrupteur n'arrive pas à couper la parole au locuteur actuel qu'après avoir parlé simultanément sur un certain nombre de syllabes. Cette dernière qui s'accompagne d'un chevauchement, a «pour tendance taxémique générale d'exprimer une position haute, ou une tentative de l'accaparer.» (KERBRATORECCHIONI, 1992, p. 88) Ces deux types d'interruptions surgissent fortement dans les débats politiques, en particulier dans le débat politique télévisé constituant notre corpus. Celui-ci a été décrit comme l'un des face à face télévisés le plus violent, le plus féroce et le plus chaotique dans l'histoire des débats politiques en France.

À l'égard des interruptions utilisées dans notre corpus, nous remarquons qu'elles sont caractérisées par la férocité et la violence mutuelles dans la mesure où le débat s'est transformé en guerre verbale. Dès le début du débat jusqu'à la fin, les deux finalistes se sont concentrés sur l'échange d'accusations, notamment de la part de Marine Le Pen qui semblait plus offensive que E. Macron. L'interruption du discours de l'autre est tout à fait attendue dans de tels débats, mais pas de cette manière agressive. Les questions qui se posent maintenant: Pourquoi les participants à ce débat

(L'interruption et le chevauchement dans le débat...) Mohamed HAMZA 
recourent-ils à ces interruptions? Comment gèrent-ils ce dysfonctionnement interactionnel? Quelle est la visée de ces interruptions? Telles questions trouveront dorénavant leurs réponses.

Nous nous contenterons ici de présenter une étude interactionnelle des interruptions dans ce débat politique. Les interruptions peuvent, dans ce cadre, être impulsées par une intention polémique ou une intention coopérative. À cela s'ajoutent les interruptions des modérateurs qui visent à bien gérer le débat. Commençons par indiquer les interruptions à fonction polémique.

\subsubsection{Interruptions à fonction polémique}

Ce sont des interruptions qui ont plus ou moins un caractère violent, agressif et vif, notamment dans notre corpus. Ce cas d'interruptions se produit «lorsqu'un locuteur essaie de prendre la parole en faisant de ses propres remarques une priorité plus élevée par rapport au discours du locuteur en place dans le cas où ce dernier a l'intention de continuer. (Notre traduction)(8)» (KUPPEVELT \& SMITH, 2003, p. 357) Telles interruptions permettent au locuteur de s'emparer de la parole en empiétant sur le locuteur en place. Cette scène compétitive peut se faire dans le but de prendre la parole, poser une question, faire un commentaire, rivaliser pour un sujet ou une idée, etc. Dans l'un ou l'autre de ces cas compétitifs, «l'interruption agit pour retirer l'intention du

(L'interruption et le chevauchement dans le débat...) Mohamed HAMZA 
locuteur principal, au moins temporairement, et la concentrer sur le discours de l'interrupteur. (Notre traduction)(9)» (KUPPEVELT \& SMITH, 2003)

Dans le corpus, il est à remarquer que les interruptions à fonction polémique sont les plus représentées parmi les autres types d'interruptions et se marquent par la brutalité et la violence lexicale. Bien que ce débat-ci se déroule en contexte électoral, chaque candidat s'efforce non seulement de clarifier son point de vue, ses idées politiques et son programme électoral, mais aussi de répliquer et de montrer les contradictions criantes de son adversaire.

Dès les premières prises de parole jusqu'à la fin du débat, E. Macron et M. Le Pen ne cessent pas de s'interrompre sur tous les dossiers: l'économie, le terrorisme, l'éducation, etc. À titre d'exemple, citons ce passage qui porte sur le dossier de la santé: 


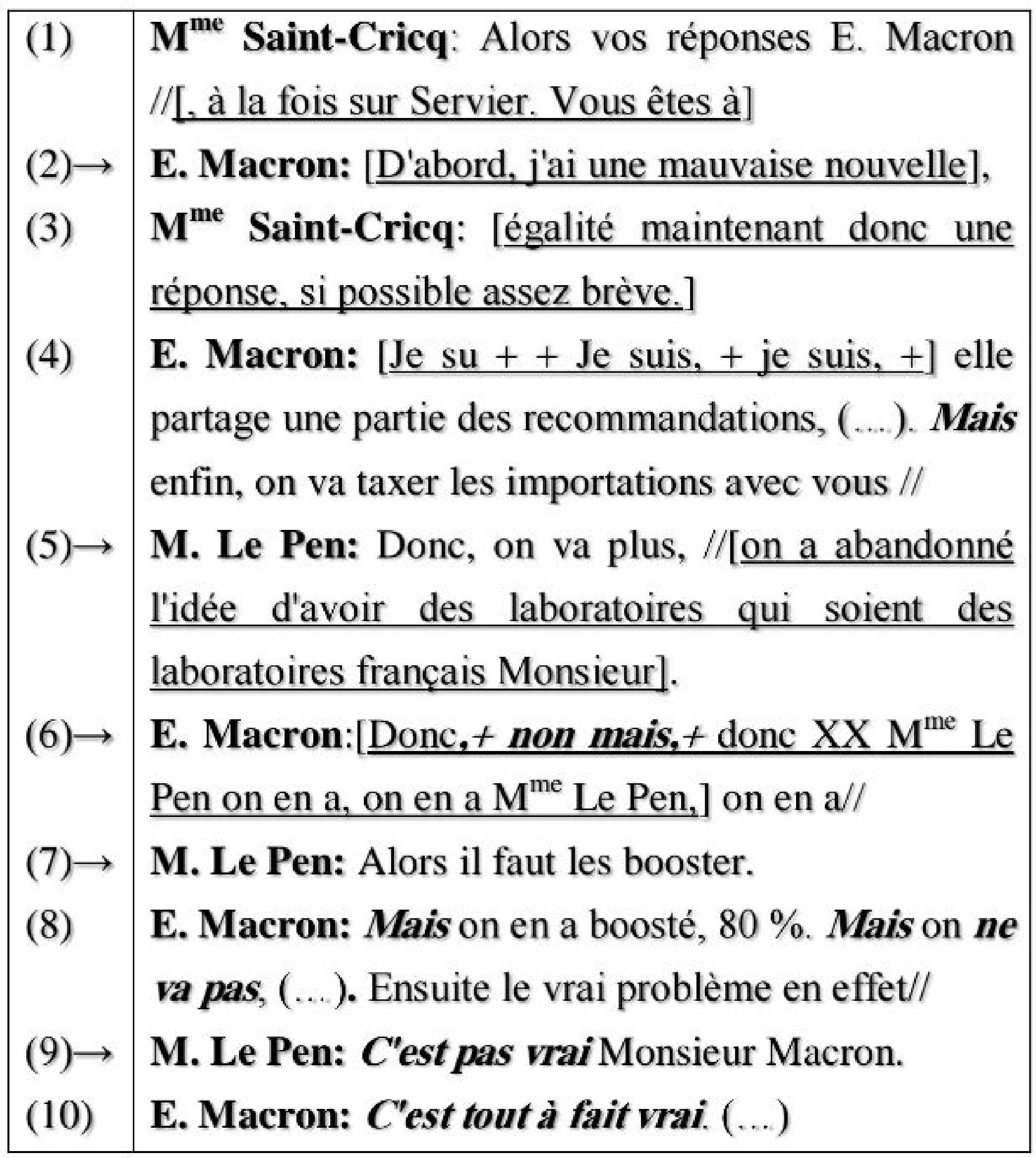

Au début de cet exemple, la modératrice donne le tour de parole à $\mathrm{E}$. Macron pour donner des réponses concernant (Les laboratoires Servier), une société pharmaceutique internationale connue. Mais en (2), le successeur potentiel "E. Macron" prend la parole trop tôt et interrompt $\mathbf{M}^{\mathrm{me}}$ Saint Cricq sans attendre la fin de son discours. Bien que ce soit le tour de parole de Macron, M. Le Pen s'empare de la parole en 
l'interrompant délibérément 3 fois à visée polémique. Par ses énoncés interruptifs, Le Pen veut insinuer qu'E. Macron est proche des (Laboratoires Servier), laissant entendre qu'il est prisonnier de cette société. En ( 1 et 2$)$, ( 3 et 4 ) ( 5 et 6 ), les interruptions s'accompagnent d'un chevauchement. Tandis que, les interruptions en (4), (6) et (8) sont nettes, c'est-àdire sans aucun chevauchement. M. Le Pen interrompt E. Macron non pour se défendre, mais pour s'opposer à son discours et attaquer sa manière de faire. E. Macron devient donc en position de défense pour contrer l'attaque de M. Le Pen. Telle opposition est bien indiquée grâce aux morphèmes négatifs (non, ne va pas) qui nient l'existence ou l'action, et à la conjonction adversative (mais) qui marque l'opposition, la réfutation et la rectification de ce qui précède. Une autre forme de cette opposition entre les deux candidats, c'est la présence de deux énoncés opposés ( $C$ 'est pas vrai / $C$ 'est tout à fait vraı).

Sur le dossier de la sécurité et du terrorisme comme d'habitude sur tous les dossiers, les échanges, entre les deux candidats combattants, ont été très tendus et pleins d'interruptions. M. Le Pen n'a pas seulement évoqué la lutte contre le fondamentalisme islamiste, mais elle a encore accusé E. Macron de courtiser ces courants et de les soutenir. On comprend donc que ces deux candidats opposés complètement se font face. Considérons ainsi cet exemple où 
M. Le Pen transgresse «le principe de qualité» (KERBRATORECCHIONI, 1990, p. 178), ce qui amène E. Macron à l'interrompre quasiment 9 fois pour rétorquer ses arguments fallacieux:

\begin{tabular}{|c|c|}
\hline (1) & $\begin{array}{l}\mathbf{M}^{\text {me }} \text { Saint Cricq: }(. . .) \text {, je voulais savoir quelles } \\
\text { sont vos propositions } M \text {. Le Pen, dans le contexte } \\
\text { actuel. + Très concrètement + }\end{array}$ \\
\hline (2) & $\begin{array}{l}\mathbf{M}^{\text {me }} \text {. Le Pen: La sécurité et le terrorisme, (...) } \\
\text { Totalement absente de votre projet, (....). Je sais } \\
\text { que vous êtes contre cela mais vous êtes pour } \\
\text { toutes les propositions laxistes et contre toutes } \\
\text { celles qui font preuve de fermeté// }\end{array}$ \\
\hline (3) $\rightarrow$ & $\begin{array}{l}\text { M. Macron: Vous me laisserez expliquer mon } \\
\text { projet Madame Le Pen, parlez plutôt du vôtre. }\end{array}$ \\
\hline (4) & $\begin{array}{l}\mathbf{M}^{\mathrm{me}} \text {. Le Pen: Et, déchus de leur nationalité ils } \\
\text { seront également } / /[\text { renvoyés dans le pays. Les } \\
\text { fichés restent]. }\end{array}$ \\
\hline (5) & $\begin{array}{l}\text { M. Macron:[Ca va leur faire peur, pour ce qui se } \\
\text { font sauter, ça va les] terroriser. Non la déchéance, } \\
(\ldots)+/ /\end{array}$ \\
\hline$(6) \rightarrow$ & $\begin{array}{l}\mathbf{M}^{\mathrm{me}} \text {. Le Pen: Donc vous attendez l'attentat, vous? } \\
\text { Bien moi, je n'attends pas l'attentat// }\end{array}$ \\
\hline$(7) \rightarrow$ & $\begin{array}{l}\text { M. Macron: Mais je n'attends pas l'attentat. (...) } \\
\text { Faut aller beaucoup plus au cœur, beaucoup plus au } \\
\text { fond, être beaucoup plus concret// }\end{array}$ \\
\hline
\end{tabular}

(L'interruption et le chevauchement dans le débat...) Mohamed HAMZA 


\begin{tabular}{|c|c|}
\hline$(8) \rightarrow$ & $\begin{array}{l}\mathbf{M}^{\text {me }} \text {. Le Pen: Monsieur, pas de leçons sur ce } \\
\text { sujet-là//. }\end{array}$ \\
\hline$(9) \rightarrow$ & M. Macron: Non pas de leçons en effet. \\
\hline (10) & $\begin{array}{l}\mathbf{M}^{\mathrm{me}} \text {. Le Pen: Done avant, }(\ldots) \mathrm{C}^{\prime} \text { est-à-dire que } \\
\text { vous vous présentez à l'élection présidentielle } / /[\underline{\mathrm{et}} \\
\text { vous n'avez même pas un programme constitué, } \\
\text { sérieux, cohérent.] }\end{array}$ \\
\hline (11) & $\begin{array}{l}\text { M. Macron: }[\approx \text { Je l'ai } \approx \text { Madame, Mais Madame } \approx \\
\text { je l'ai } \approx \text {. Mais je ne vais pas tomber comme vous } \\
\text { dans le } \approx \text { piège } \approx] \text { des sauts de cabri. }\end{array}$ \\
\hline (12) & $\mathbf{M}^{\mathrm{me}}$. Le Pen: Vous pouvez me laisser terminer? // \\
\hline$(13) \rightarrow$ & $\begin{array}{l}\text { M. Macron: Mais non, parce que constamment } \\
\text { vous êtes }(\ldots)\end{array}$ \\
\hline (14) & $\begin{array}{l}\mathbf{M}^{\mathrm{me}} \text {. Le Pen: Vous êtes très énervé. } / /[\underline{\text { Laissez-moi }} \\
\text { parler }] \text {, }\end{array}$ \\
\hline (15) & M. Macron: [Je ne suis pas du tout énervé], \\
\hline (16) & $\mathbf{M}^{\mathrm{me}}$. Le Pen: Regardez! j'ai du retard sur vous// \\
\hline$(17) \rightarrow$ & $\begin{array}{l}\text { M. Macron: Madame Le Pen! Je sais et vous allez } \\
\text { le rattraper. }\end{array}$ \\
\hline (18) & $\mathbf{M}^{\mathrm{me}}$. Le Pen: Alors laissez-moi parler// \\
\hline$(19) \rightarrow$ & $\begin{array}{l}\text { M. Macron: Mais parlez de votre projet et ne dites } \\
\text { pas de bêtises sur le mien. (....) }\end{array}$ \\
\hline
\end{tabular}

Dans ce passage, $\mathbf{M}^{\mathrm{me}}$ Saint Cricq adresse la parole à M. Le Pen et lui demande, selon les règles du débat, d'assumer le discours sur la sécurité et le terrorisme. Mais en 
(2), M. Le Pen commence bientôt à attaquer le projet de son adversaire en l'accusant de courtiser et de soutenir le fondamentalisme islamiste. C'est ce qui excite E. Macron et le conduit, en (3), à commettre une sorte de violation territoriale en l'interrompant plusieurs fois pour défendre son projet. En position de défense, Macron recourt à des énoncés interruptifs introduits soit par des adverbes négatifs ( Non, Non pas, pas de) soit par l'emploi de (mais) en contexte réfutatif. $\mathrm{Ce}$ (mais) est accompagné de la négation qui est nécessaire à l'interprétation de "mais" de réfutation. L'emploi de telle structure «articule des arguments antiorientés et introduit un conflit de parole.» (LOPEZ-MUŇOZ, MARNETTE, \& ROSIER, 2005, p. 170) On est alors devant deux visions tout à fait opposées, étant donné que les deux candidats se disputent sur la déchéance de la nationalité pour tous ceux qui sont double nationaux. Pour cela, nous trouvons qu'il y a un flot d'interruptions, suivant les stratégies défense-attaque, soit avec chevauchement en (4 et 5) (10 et 11) (14 et 15) ou sans chevauchement en $(3,6,7,8,9,13,17,18)$. La présence des énoncés, comme ("Laissez-moi parler", "Vous pouvez me laisser terminer?", "Alors laissez-moi parler!"), exprime la sensation de M. Le Pen de s'être fait couper la parole. D'autre part, la présence de l'énoncé (ne dites pas de bêtises sur le mien.) résume pourquoi $\mathrm{E}$. Macron recourt à ces interruptions de parole. Ce

(L'interruption et le chevauchement dans le débat... ) Mohamed HAMZA 
dernier s'en sert en effet pour remettre les choses en place et répondre aux arguments fallacieux de $\mathrm{M}$. Le Pen.

En regardant ces deux extraits du débat télévisé, constituant les deux exemples cités ci-dessus, nous remarquons que les énoncés interruptifs à fonction polémique sont accompagnés des signaux: soit verbaux (intonation ascendante, accentuation syllabique et allongement syllabique), soit paraverbaux comme les mimiques faciales et les gestes (regard, levage les mains et pointage les doigts). Ces signaux traduisant clairement la colère et les réactions de chaque candidat donnent aux énoncés interruptifs un caractère violent et agressif.

Après avoir abordé les interruptions à fonction polémique, il est à remarquer qu'elles sont les plus représentées tout au long du débat. C'est peut-être parce que l'ensemble du débat porte sur l'attaque, la défense et l'argumentation de la part de chaque candidat. Mais, ce qui attire aussi notre attention dans ce débat, c'est la stratégie de guerre totale à laquelle chaque candidat recourt. Cette stratégie consiste à bombarder l'adversaire sans relâche afin d'objecter ses propositions ainsi que son point du vue. Néanmoins, les interruptions s'orientent parfois vers le soutien du locuteur en place. Tel sera notre point d'analyse ci-après.

(L'interruption et le chevauchement dans le débat...) Mohamed HAMZA 


\subsubsection{Interruptions à fonction coopérative}

C'est le cas où les interruptions visent à soutenir le locuteur en place. Il s'agit des interruptions destinées à aider le locuteur principal en coordonnant le processus ou le contenu de l'interaction en cours soit par des commentaires laconiques, soit par un soutien linguistique ou par des questions de clarification. C'est ce qui amène J. V. Kuppevelt et $\mathrm{R}$. W. Smith à dire que les interruptions coopératives «se produisent lorsqu'un locuteur veut appuyer ou renforcer le point soulevé par le locuteur en cours sans perturber la continuation de ce locuteur. Ces types de remarques de soutien prennent souvent la forme de brefs commentaires ou de questions de clarification. De telles questions de clarification soutiennent souvent le flux continu de locuteur en cours en gardant les deux locuteurs en synchronie sur le développement du sujet. (Notre traduction)(10)» (KUPPEVELT \& SMITH, 2003, p. 357)

De telles interruptions sont dirigées vers la relation collaborative et garantissent le bon déroulement de l'interaction. Elles expriment l'intérêt enthousiaste et l'implication active dans le discours, d'autant plus que $\mathrm{C}$. Kerbrat-Orecchioni le trouve comme «manifestation empressée d'un accord ou d'une adhésion ( $L_{2}$ apporte en enthousiasme de l'eau au moulin de $\mathrm{L}_{1}$ ), ou tout simplement marque d'une participation active et d'une implication intense

(L'interruption et le chevauchement dans le débat...) Mohamed HAMZA 
dans l'échange communicatif.» (KERBRAT-ORECCHIONI, 1990, p. 178)

Dans le corpus, les occurrences, relevant de l'objectif coopératif, sont très peu fréquentes et s'utilisent de temps en temps par les participants. Ces cas sont représentés sous la forme d'un soutien linguistique, d'un accord momentané ou d'une question de clarification. Par rapport au premier cas d'assistance, l'interrupteur «perçoit que le locuteur en place a besoin d'aide. L'interrupteur fournit donc au locuteur en place un mot, une phrase, une locution ou Idée. (Notre traduction)(11)» (LI, 2001, p. 269) Prenons alors la seule occurrence qui représente le soutien lexical:

\begin{tabular}{|l|l|}
\hline$(1)$ & $\begin{array}{l}\mathbf{M}^{\text {me }} \text {. Le Pen: Vous savez, (...) Vous avez parlé } \\
\text { de la France en disant: «La France sera respectée } \\
\text { si elle est une grande puissance économique, etc.» } \\
\text { ça y est, on revient au graphique // au budget } \\
\text { M. Macron: et politique }\end{array}$ \\
\hline
\end{tabular}

M. Le Pen développe sa vision sur la nécessité, pour la France, de conserver son identité, de retrouver son indépendance et de ne pas soumettre à la vision impérialiste. Tout en critiquant la vision de Macron, elle évoque ironiquement ce que Macron a dit plus tôt que la puissance de la France réside dans une économie forte, mais elle oublie une partie de son discours. Immédiatement, E. Macron décide de l'interrompre pour l'aider linguistiquement et lui

(L'interruption et le chevauchement dans le débat...) Mohamed HAMZA 
rappeler le mot (et politique). Cet énoncé interruptif est apparemment à visée collaborative, mais elle est en effet teintée de polémique visant à défendre sa vision. Cet énoncéci s'accompagne d'une intonation aigue et de signaux mimogestuels (le regard, secouer la tête et lever les sourcils) qui expriment le ressentiment de la part de Macron.

S'agissant des interruptions coopératives, exprimant l'accord momentané entre les deux candidats, elles sont rarement représentées lors du débat. C'est peut-être parce que les deux finalistes, appartenant à deux tendances politiques totalement contradictoires, ont deux visions complètement irréconciliables: E. Macron est un libéral et pro-européen, alors que M. Le Pen est isolationniste et anti-européenne. Remarquons cet exemple où l'accord est seulement momentané:

\begin{tabular}{|c|c|}
\hline (1) & $\begin{array}{l}\text { M. Macron: (...) Alors qu'on devrait développer } \\
\text { l'implantation des spécialistes sur la totalité } / /[\underline{\text { du }} \\
\text { territoire francais }] \text {. }\end{array}$ \\
\hline$(2) \rightarrow$ & $\begin{array}{l}\mathbf{M}^{\mathrm{me}} \text { Le Pen: [Monsieur Macron, il y a surtout un } \\
\text { énorme] problème d'immigration clandestine et } \\
/ /[\text { le système de santé est noyé par l'immigration } \\
\text { clandestine en Guyane et à Mayotte]. }\end{array}$ \\
\hline$(3) \rightarrow$ & $\begin{array}{l}\text { M. Macron: }+[\mathrm{xx} \text { On y reviendra. Mais euh euh } \\
+\mathrm{y} \text { a aussi, y a }] \text { y a aussi un problème } \\
\text { d'immigration, vous avez tout à fait raison, }(. . .)\end{array}$ \\
\hline
\end{tabular}

(L'interruption et le chevauchement dans le débat...) Mohamed HAMZA 
En (1), E. Macron prend son tour de parole pour exprimer la situation critique par rapport au système de santé et les solutions proposées selon son projet. En (2) et (3), M. Le Pen et E. Macron s'interrompent mutuellement avec un chevauchement assez long et une intonation assez forte de la part de Le Pen. En (2), M. Le Pen s'accorde implicitement avec lui par rapport aux problèmes du système de santé, notamment à Guyane et à Mayotte. En (3), Macron est pour cet avis et soutient complètement ce que M. Le Pen dit. En exprimant son approbation, Macron recourt à la reformulation de ce que $\mathrm{M}$. Le Pen a mentionné à l'aide de l'adverbe (aussi) et l'énoncé (vous avez tout à fait raison) qui traduit l'accord intégral entre les deux. A cela s'ajoute l'utilisation d'une intonation intermédiaire appuyée par un geste de main affirmant cet accord.

Les interruptions coopératives pourraient encore s'utiliser, comme on vient de le dire, pour une demande de clarification. Dans ce cas, l'interrupteur coupe la parole au locuteur en place avec l'intention de demander des éclaircissements sur le sujet à traiter. Par conséquent, «le but ultime de l'interruption est de demander au locuteur en place de clarifier ou d'expliquer une partie d'informations précédemment obtenue qui n'est pas claire pur l'auditeur. (Notre traduction)(12)» (LI, 2001, p. 269) Dans le corpus, ces interruptions expriment apparemment la demande de

(L'interruption et le chevauchement dans le débat... ) Mohamed HAMZA 
clarification et soutiennent le flux continu de locuteur en cours. Mais, elles sont effectivement un moyen de déstabiliser l'adversaire et de montrer les points faibles de son projet comme le montre cet exemple:

\begin{tabular}{|c|c|}
\hline (1) & $\begin{array}{l}\mathbf{M}^{\text {me }} \text { Le Pen: Juste avant que l'euro devienne notre } \\
\text { monnaie// }\end{array}$ \\
\hline$(2) \rightarrow$ & M. Macron: Ah bon?! \\
\hline (3) & $\mathbf{M}^{\text {me }}$ Le Pen: physique en France. Mais oui, //+ \\
\hline$(4) \rightarrow$ & M. Macron: C'était quoi? \\
\hline (5) & $\begin{array}{l}\mathbf{M}^{\text {me }} \text { Le Pen: mais oui, mais oui. C'était l'euro déjà } \\
\text { et //[avant lui c'était l'écu]. }\end{array}$ \\
\hline$(6) \rightarrow$ & $\begin{array}{l}\text { M. Macron: }[\text { Donc }+ \text {, donc, }] \text { donc les grandes } \\
\text { entreprises, elles payaient en euro et en écu avant?! }\end{array}$ \\
\hline (7) & $\begin{array}{l}\mathbf{M}^{\text {me }} \text { Le Pen: Mais oui, de } 1993 \text { à } 2002 \text {, toutes les } \\
\text { entreprises pouvaient payer// }\end{array}$ \\
\hline$(8) \rightarrow$ & M. Macron: $\approx$ D'accord! $\approx$ \\
\hline
\end{tabular}

Dans ce passage, M. Le Pen a évoqué le système de l'ECU comme modèle pour la "monnaie commune" qu'elle compte instaurer au lieu de l'euro. C'est ce qui conduit E. Macron à l'interrompre intentionnellement plusieurs fois en vue de poser des questions de clarification sur ce système monétaire. Ces questions, surtout les interrogations totales en (2) et (4), sont teintées d'étonnement et accompagnées d'une montée intonative à la finale de l'énoncé interrogatif et d'expressions faciales (yeux ouverts et sourcils levés). Les 
marques d'étonnement que Macron a suscitées, en posant ces questions, sont interactivement et discursivement significatives. Macron veut prouver aux téléspectateurs que ce système monétaire est imaginaire et irréaliste, particulièrement après qu'il ait multiplié, sur le même sujet, les questions à l'adversaire. Mais en (5), l'énoncé interruptif (d'accord), accompagné d'un allongement syllabique, exprime sémantiquement l'accord de Macron avec Le Pen, mais il signifie pragmatiquement selon la situation de communication (enfin, j'ai à peine compris ce que vous disiez)

Après l'examen des interruptions à fonction coopérative entre les deux finalistes, nous pouvons constater qu'elles sont très peu nombreuses dans leur stratégie communicative. Cela peut-être tient à la nature de ce débat très violent qui présente deux projets tout à fait opposés. Ce débat comprend, outre les interruptions des candidats, d'autres interruptions de la part des modérateurs. C'est sous cet angle que nous allons analyser les interruptions des modérateurs et leur objectif au cours de la discussion.

\subsubsection{Interruptions des modérateurs}

Bien connu que les modérateurs jouent un rôle primordial à préserver la cohérence globale du débat. Ils prennent en charge la régie du débat. Par conséquent, ils effectuent plusieurs tâches comme: gérer et contrôler les 
tours de parole, sélectionner les questions à poser, lancer les sujets à discuter, veiller l'égalité du temps de parole entre les candidats, etc. Compte tenu de ce rôle interactionnel, les modérateurs ont le pouvoir institutionnel d'interrompre les personnes interrogées dans le cas où ces dernières transgressent l'une des règles déjà fixxées qui régissent le débat. Bien que les modérateurs s'efforcent de prendre des décisions importantes de gestion, ils «doivent peser / réfléchir s'il est bénéfique pour les téléspectateurs qu'ils interrompent les interventions des candidats ou pas.» (MARINESCU, 2017, p. 37)

Dans ce débat présidentiel, les interruptions des modérateurs visent le plus souvent à bien régir cette confrontation. Ces interruptions intentionnelles ont précisément pour objectifs de gérer les tours de parole, les thématiques, le temps entre les deux concurrents et poser une question de clarification. Les interruptions, relevant de ces objectifs, sont toutes présentes dans le corpus. Concernant l'interruption qui a pour objectif de gérer les tours de parole, le modérateur interrompt un candidat pour lui indiquer la fin de son tour et/ou pour donner la parole à l'autre candidat. Examinons alors cet exemple:

(L'interruption et le chevauchement dans le débat... ) Mohamed HAMZA 


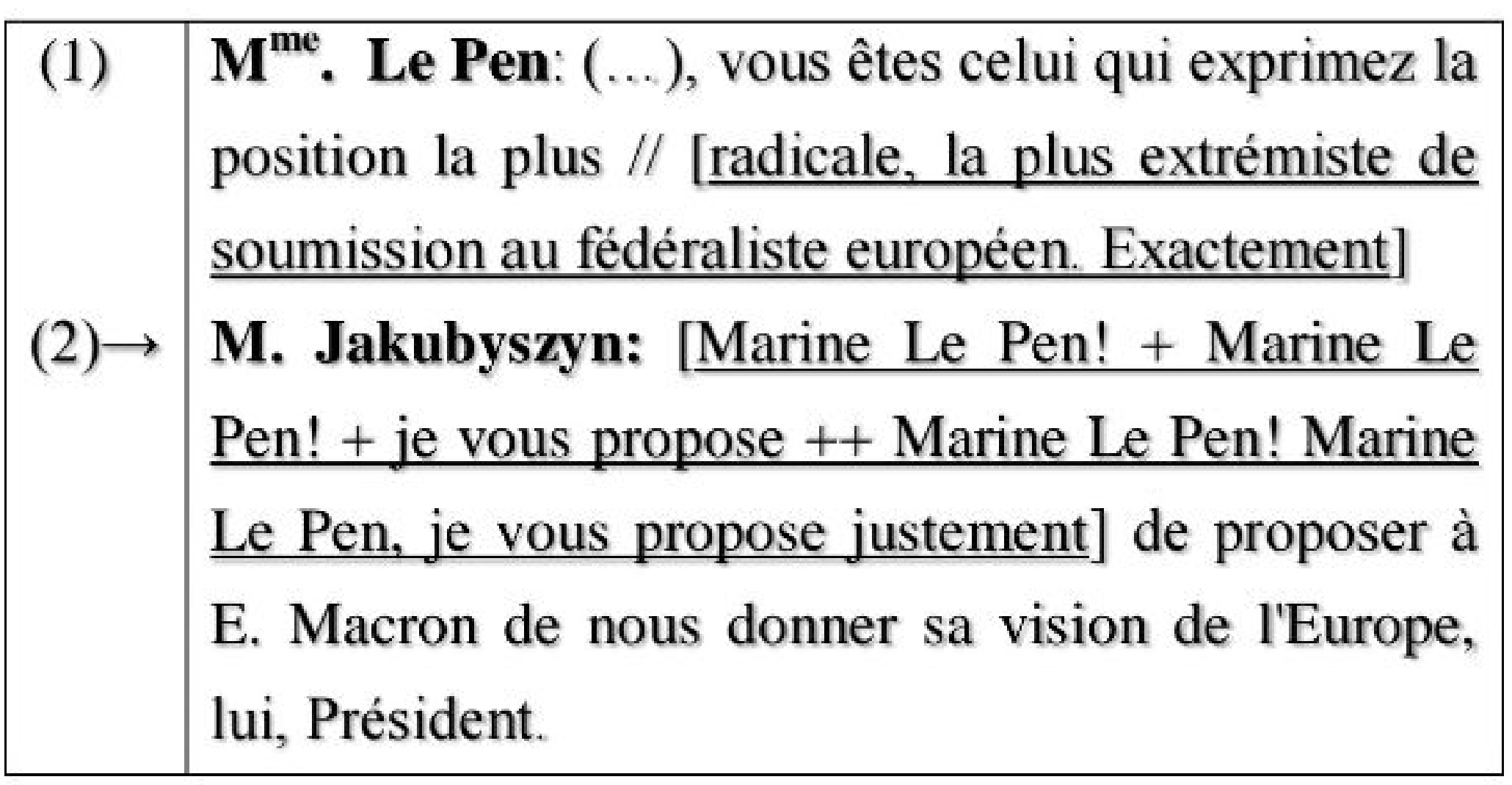

Cet exemple illustre comment le modérateur coupe gentiment la parole à $\mathrm{M}$. Le Pen en lui proposant de donner le tour de parole à E. Macron. Pour y parvenir, le modérateur recourt à répéter "Marine Le Pen" 4 fois, c'est ce qui montre comment il prend difficilement la parole. Cet énoncé interruptif délibéré est done accompagné d'un chevauchement à peu près 8 secondes. Ce discours chevauchant est conduit d'une intonation ascendante de la part de $\mathrm{M}$. Le Pen. Une telle intonation de cette dernière reflète l'insistance à ne pas laisser la parole jusqu'à ce qu'elle termine complètement ce qu'elle veut dire comme le traduit l'emploi de l'adverbe (exactement).

Quant aux interruptions touchant la gestion des thématiques, elles sont particulièrement exécutées par les modérateurs. Ceux-ci sont obligés de respecter un programme thématique préconçu portant sur le développement d'un certain nombre de sujets qui intéressent 
énormément les Français. À cet effet, l'un des modérateurs interrompt le locuteur en cours tantôt en vue de lancer un nouveau thème à discuter, tantôt en vue de lui rappeler le thème dont il doit parler. A titre d'exemple, citons ce passage:

\begin{tabular}{|l|l|}
$(1)$ & $\begin{array}{l}\mathbf{M}^{\mathrm{me}} \text {. Le Pen: Tous //quasiment + on savait avant } \\
\text { tous //++ tous Monsieur } \\
\mathbf{M}^{\mathrm{me}} \text { Saint-Cricq: Madame Le Pen! ++ Madame } \\
\text { Le Pen! + on nous accuse de pas parler de fond, de } \\
\text { temps en temps // [et là un sujet ++ un sujet +qui } \\
\text { préoccupe les Français]... } \\
(3) \rightarrow\end{array}$ \\
$(4) \rightarrow$ & $\begin{array}{l}\mathbf{M}^{\mathrm{me}} \text { Le Pen: [C'est très important le Premier } \\
\text { ministre]. } \\
\mathbf{M} . \text { Macron: [C'est très important XX sur la } \\
\text { retraite] on reste sur un système de répartition qui } \\
\text { est plus juste et plus transparent //et qui est, lui, } \\
\text { financé //contrairement aux autres. } \\
\mathbf{M}^{\text {me }} \text { Saint-Cricq: Alors ++ très bien + alors à } \\
\text { propos de sécurité, terrorisme, c'est quand même } \\
\text { un sujet qui préoccupe énormément les Français. }\end{array}$ \\
\hline
\end{tabular}

En (2), la modératrice s'empare de la parole interrompant M. Le Pen pour lui demander de ne pas s'éloigner du sujet à traiter. Puis en (2), (3) et au début de (4), la modératrice et les deux candidats s'interrompent avec un peu de chevauchement de parole. Celui-ci est suivi d'une 
intonation forte de la part de Macron, c'est ce qui lui permet finalement de monopoliser la parole. Mais en (5), la modératrice reprend difficilement la parole après avoir coupé la parole à Macron annonçant le nouveau sujet. Ce qui est plus remarquable dans ce passage, c'est le manque d'autorité de la modératrice sur le plateau du débat. L'hésitation et le recourt à la répétition accompagnée des pauses affïrment ce manque d'autorité.

En fait, la répartition de la parole doit être motivée par des contraintes qui garantissent l'égalité du temps de parole entre les candidats. Pour ce faire, les modérateurs coupent parfois la parole au locuteur en place afin de régir le temps de parole: soit pour l'informer de la fin du temps de parole, soit pour lui rappeler le temps restant. Considérons ainsi l'exemple subséquent:

\begin{tabular}{|l|l|}
\hline$(1)$ & $\begin{array}{l}\mathbf{M}^{\text {me }} \text {. Le Pen: (...) C'est pour ça que vous êtes allé } \\
\text { en Algérie nous vendre une autoroute migratoire // } \\
\text { [entre la France] + euh euh euh }\end{array}$ \\
$(2) \rightarrow$ & M. Jakubyszyn: [vous avez trente secondes.] \\
\hline
\end{tabular}

Ce passage se situe à la fin du débat où les deux modérateurs essaient de régler les conclusions dont le temps déterminé est deux minutes. Ce qui conduit le modérateur à interrompre soudainement $\mathrm{M}$. Le Pen afin de lui rappeler que son tour touche à sa fìn. Cette interruption est intentionnelle et s'accompagne d'un chevauchement très court. L'utilisation

(L'interruption et le chevauchement dans le débat...) Mohamed HAMZA 
de l'interjection (euh) trois fois successives suggère que cet énoncé interruptif fait hésiter $M$. Le Pen qu'elle récupère difficilement la parole.

Les interruptions, visant à poser une question de clarification sur le thème à aborder, sont une stratégie à laquelle a le plus souvent recours l'un des candidats afin de déstabiliser et confondre son adversaire. Pour autant, les modérateurs s'en servent épisodiquement à la gestion du débat en cas de nécessité pour clarifier certains points nécessaires:

\begin{tabular}{|l|l|}
$(1)$ & $\begin{array}{l}\mathbf{M}^{\text {me }} \text { Le Pen: (...) Faire parallèlement un } \\
\text { référendum constitutionnel que je soumettrai aux } \\
\text { Français// } \\
\mathbf{M}^{\text {me }} \text { Saint-Cricq: On a une idée de quand? } \\
\mathbf{M}^{\text {me }} \text { Le Pen: Le référendum constitutionnel sera en } \\
\text { septembre, précisément en septembre, (...). (...) et } \\
\text { donc il faut immédiatement //[prendre cette } \\
\text { décision], immédiatement prendre cette décision. } \\
\text { M. Jakubyszyn: On va en parler plus précisément } \\
\text { (3) } \rightarrow \\
\text { tout à l'heure. } \\
\mathbf{M}^{\text {me }} \text { Le Pen: (...), bien je leur demanderai leur avis } \\
/ /[\text { et bien entendu }+ \text { je respecterai cet avis] } \\
\mathbf{M}^{\text {me }} \text { Saint-Cricq: [Ça c'est dans combien de temps } \\
\text { cet autre référendum? Vous donnez] combien de } \\
\text { temps? } \\
\mathbf{M}^{\text {me }} \text { Le Pen: Ecoutez, j'ai dit six mois, (....) }\end{array}$ \\
\hline (7)
\end{tabular}

(L'interruption et le chevauchement dans le débat...) Mohamed HAMZA 
L'analyse porte sur les énoncés interruptifs $(2,6)$. Au début M. Le Pen présente sa vision sur l'Europe de demain en répondant à la question qui lui était déjà posée et comment elle voit la nécessite de la sortie de l'euro en vue de restituer au peuple français sa souveraineté (monétaire, législative, territoriale ....). Mais en (2), la modératrice lui coupe la parole sans aucun chevauchement pour lui demander une clarification sur un point particulier (le moment de ce référendum constitutionnel). En (6), la modératrice l'interrompt à nouveau, mais cette fois avec chevauchement, en lui demandant des précisions sur (la durée de l'autre référendum). Les deux énoncés interrogatifs se terminent sur une intonation ascendante, soutenue par un geste de doigt indiquant une demande de réponse précise. De telles interrogations apparaissent légitimes pour les modérateurs et correspondent à leur rôle interactionnel.

Après avoir abordé les interruptions touchant à la gestion du débat, nous constatons qu'elles sont employées par les modérateurs et coïncident exactement avec le rôle interactionnel de ces derniers. Bien que ces interruptions délibérées soient vues comme des dysfonctionnements interactionnels, elles sont de temps en temps indispensables pour mener à bien leur travail. Par autant, nous remarquons, en analysant ce point, que les deux modérateurs n'ont pas de rôle prédominant de bien gérer le débat sur le plateau. Ils sont

(L'interruption et le chevauchement dans le débat... ) Mohamed HAMZA 
hésitants, presque inaudibles ou absents devant les échanges brouillons des deux candidats combattants.

\section{- Conclusion}

Cet article s'est concentré sur l'analyse de l'interruption et du chevauchement comme des ratés du système des tours dans un débat politique télévisé décrit comme un combat verbal violent. Ce débat inouĩ a présenté un échange musclé entre deux finalistes prêts à en découdre. En examinant le système des tours de parole suivi conformément aux règles médiatiques régissant ce débat, il est à remarquer qu'il reste seulement respecté pendant 5 minutes et 14 secondes du début du débat soit par les deux finalistes, soit par les modérateurs. Après cette période, la guerre verbale a commencé entre les candidats au point que les modérateurs ne pouvaient pas contrôler le débat.

Le chevauchement et l'interruption sont des phénomènes discursifs liés et correspondent à différentes manières pour un locuteur qui intervient sur la parole d'un autre. Ces deux phénomènes langagiers sont, dans le débat, réalisés séparément ou conjointement et accompagnés des signaux spécifiques (prosodiques et mimo-gestuels) qui sont analysés conformément au contexte. Les deux finalistes y ont recouru soit à fin polémique, soit à fin coopérative. Néanmoins, les chevauchements et les interruptions à fin polémique sont les plus représentés tout ou long du débat et

(L'interruption et le chevauchement dans le débat... ) Mohamed HAMZA 
se marquent par la brutalité, la cacophonie et la violence lexicale. Que ce soit E. Macron ou M. Le Pen, chacun s'efforce non seulement de se défendre, de clarifier son point de vue, ses idées politiques et son programme électoral, mais aussi de critiquer, répliquer et déstabiliser l'adversaire.

Il y a également des interventions effectuées par les modérateurs qui se cristallisent encore sous la forme des chevauchements et des interruptions visant à gérer le débat: contrôler la progression thématique, contrôler le temps de parole, intervenir lorsque la cacophonie empêche la compréhension, etc. $\mathrm{Ce}$ qui attire notre attention, en examinant ces interventions, c'est le manque d'expérience des modérateurs à bien gérer le débat. Ils sont presque inaudibles et parfois absents devant les échanges désordonnés et turbulents des deux candidats. L'hésitation, la voix faible et le recourt à la répétition accompagnée des pauses affirment le manque d'autorité sur le plateau. Ce manque d'autorité est l'une des principales raisons du désordre et de la violation du système des tours qui ont dominé ce débat.

Nous avons tenté de jeter la lumière sur les ratés du système des tours à travers l'étude des phénomènes interactionnels - l'interruption et le chevauchement - qui contrecarrent le principe d'alternance des tours de parole.

(L'interruption et le chevauchement dans le débat...) Mohamed HAMZA 


\section{- Bibliographie}

\section{I- Références en français et en anglais}

- BEAL Christine, (2010), Les interactions quotidiennes en français et en anglais: de l'approche comparative à l'analyse des situations interculturelles, Berne, Peter Long.

- DANLER Paul et al, (2010), Actes du XXVe Congrès International de Linguistique et de Philologie Romanes: Discours d'ouverture. T. 1, Volumes 1-7, Berlin, De Gruyter.

- Florence OLOFF, (2008), "La répétition suite au chevauchement: analyse comparative de données vidéo", $\underline{\underline{i n}}$ Cahiers de praxématique, $\mathrm{n}^{\circ} 50$.

- GREVISSE Maurice et DA SILVA-GENEST Christine, (2018), Le Grevisse de l'orthophoniste: Grammaire essentielle, Louvain-la-Neuve, De Boeck Supérieur.

- GUEDJ Jean-Paul, (2008), $\underline{\underline{50} \text { fiches de }}$ communication: concepts et pratiques. techniques de management, Paris, Bréal.

- JEFFERSON Gail, (2004), "A sketch of some orderly aspects of overlap in natural conversation", Lerner G. H. (ed.), In: Conversation Analysis. Studies from the first generation, Amsterdam / Philadelphia: Benjamins.

- KERBRAT-ORECCHIONI Catherine, (1990), Les interactions verbales, tome 1, Paris, A. Colin.

(L'interruption et le chevauchement dans le débat...) Mohamed HAMZA 
- KERBRAT-ORECCHIONI Catherine, (1992), $\underline{\underline{\text { Les }}}$ interactions verbales, tome 2, Paris, A. Colin.

- KERBRAT-ORECCHIONI Catherine, (1996), $\underline{\underline{L a}}$ conversation, Paris, Seuil.

- KUPPEVELT Jan Van et W. SMITH Ronniye, (2003), Current and New Directions in Discourse and Dialogue, Dordrecht, Springer Science+Business Media.

- LI Han, (2001), "Cooperative and intrusive interruptions in inter- and intracultural dyadic discourse", in: Journal of language and social psychology, 20 (3).

- LOPEZ-MUŇOZ Juan-Manuel et al (2005), Dans la jungle des discours: genre de discours et discours rapporté, Cádiz, UCA.

- MARINESCU Valentina et BRANEA Silvia, (2017), Exploring Political and Gender Relations: New Digital and Cultural Environments, Cambridge Scholars Publishing.

- MONDADA Lorenza, (2017), "Nouveaux défis pour l'analyse conversationnelle: l'organisation située et systématique de l'interaction sociale", $\underline{\text { in. Langage et société, }}$ $\mathrm{N}^{\circ} 160-161$.

- NOSSITER Adam, "Le Pen and Macron Clash in Vicious Presidential Debate in France", In: The New York Times, article publié le 3 mai 2017.

(L'interruption et le chevauchement dans le débat...) Mohamed HAMZA 
- PLETY Robert, (1993), Ethologie des communications humaines: aide-mémoire méthodologique, Lyon, Presses Universitaires Lyon.

- PSCHAID Priska, (1993), Language and Power in the Office, Tübingen, Gunter Narr Verlag.

- SACKS Harvey et al, (1974), "A Simplest systematics for the organization of turn-taking for conversation", $\underline{\underline{I n}}$ : Language, V. 50 (4).

- SANDRÉ Marion, (2011), "Mimiques et politique. Analyse des rires et sourires dans le débat télévisé", $\mathrm{n}^{\circ}$ 96, $\underline{\underline{\mathrm{In}} \text { : Mots. }}$ Les Langages du politique.

- SCHEGLOFF Emanuel, (1987), "Recycled turn beginnings: A precise repair mechanism in conversation's turn-taking organisation", In: Talk and Social Organization, Button G., Lee J. R. E. (eds.), Clevendon: Multilingual Matters.

- SCHUlzOVA Olga, (1990), "Les procédés paralinguistiques de l'énonciation en langue slovaque", $\underline{\underline{I n}}$ : Revue des études slaves, tome 62, fascicule 1-2.

- SIDNELL Jack et STIVERS Tanya, (2013), The Handbook of Conversation Analysis, Hoboken, WILEY BLACKWELL.

\section{Sitographie}

- www.youtube.com/watch?v=i5aqL7FBxyI, consulté du (1/12/2019) jusqu'au (15/6/2020)

(L'interruption et le chevauchement dans le débat...) Mohamed HAMZA 
- www.youtube.com/watch? $\mathrm{v}=\mathrm{iOAbBdlWgz0}$, consulté du $(1 / 12 / 2019)$ jusqu'au $(15 / 6 / 2020)$

- www.vie-publique.fr/discours/203174-debat-televise-entre-emmanuel-macron-et-mme-marine-le-pen-candidats, consulté du (1/12/2019) jusqu'au (15/6/2020)

- www.nytimes.com/2017/05/03/world/europe/marine-le-enemmanuel-macron-france-presidentialdebate.html?rref=collection $\% 2$ Fsectioncollection\%2Feurope, consulté le (29/2/2020)

- Dictionnaire de français Larousse, disponible sur le site: https:/www.larousse.fr/dictionnaires/francais/interruption/43 $\underline{845}$, consulté le (10/3/2020) 


\section{- $\underline{\underline{\text { Annexe }}}$}

Voici les citations anglaises traduites en français au sein de l'article:

1-It was a study in violent verbal combat: The two talked angrily over each other, cut each other off, shook fists and pointed fingers, leaving the moderators bewildered and helpless.

2-Both interlocutors seem to strive for the floor and depending on who is the stronger, one interlocutor finally manages to get the turn.

3-Beginnings of turns are also a common site of overlapping talk.

4- Overlaps initiated in mid-turn position are not the product of turn-taking miscues. Rather, their onset appears to show next speakers' orientations to such concerns as the current turn's content, adequacy and progressivity.

5-(...) a self-selecting next speaker who orients to becoming the 'first starter' often begins a next turn slightly before the completion of the current turn, and thus winds up in brief overlap with the current speaker. Such terminal overlaps are typically fleeting since their onset is placed at a point where the current turn's completion is imminent.

6-Sometimes a brief, sometimes a longer, repeat or re-saying or recycling of part of the utterance occurs in a conversational turn.

(L'interruption et le chevauchement dans le débat...) Mohamed HAMZA 
7-This procedure appears to have some regularity of occurrence after competitive overlap. One of the competing parties announce troubles and explicitly initiates repair procedures by requesting a repeat of his co-competitor's overlapped utterance.

8-Competitive interruptions occur when one speaker attempts to take the floor by making his or him own remarks a higher priority over the main speaker's speech when the main speaker intends to continue.

9-In either of these competitive cases, interruption acts to take the intention away from the main speaker, at least temporarily and focus it on the interrupter's speech.

10- Cooperative interruptions occur when one speaker wants to support or reinforce the main speaker's point without disrupting the main speaker's continuation. These types of supportive remarks are often in the form of short commentaries or clarifying questions. Such clarifying questions often support the continuing flow of the main speaker by keebing both speakers in synchrony on the topic development.

11- The interrupter perceives that the speaker needs help. The interrupter provides the current speaker with a word, phrase, sentence, or idea.

(L'interruption et le chevauchement dans le débat... ) Mohamed HAMZA 
مجلة كلية الآداب للغويات والثقافات المقارنة

12- The ultimate goal of the interruption is to have the current speaker clarify or explain a previously elicited piece of information that the listener is unclear about. 
المقاطعة والتشابك في المناظرة السياسية المتلفزة بين جولتي الانتخابات

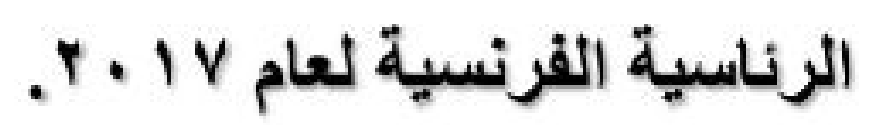

ملخص

يهذف هذا البحث إلى تثبع التجاوزات الثي ارتكبها المشاركون خلال

المناظرة السياسية المتلفزة بين جولثي الانتخابات الرئاسية الفرنسية لعام VI. Y

وما نتج عن ذلك من خلل وظيفي ثفاعلي في نظام أدوار الكلام، كما يركز البحث

علي الطريقة التي يتدخل بها المشاركون ويستولوا بها علي الكلام، لذلك فهو تحليل للمقاطعات وحالات الثشابك في الكلام خلال هذه المناظرة. ولتحقيق ذلك، نسلط الضوء أولاً على نظام أدوار الكلام ومبدأ ثناوبه والقواعد التي تثبع لضمان الأداء المثالي لعملية الثفاعل. كما نركز على المقاطعات وحالات الثشابك في الكلام و التي تكون ذات أغراض مثنوعة. ثم نحاول، من خلال تحليل مقتطفات مختلفة من المناظرة، أن نظهر أسباب استخدام هذه السلوكيات التخاطبية من قبل مختلف المشاركين وما هي الأدوار التي يمكن أن تلعبها في مناظرة سياسية متلفزة وصفت بأنها الأكثر عنفا وشراسة في تاريخ المناظرات السياسية في فرنسا.

\footnotetext{
(L'interruption et le chevauchement dans le débat...) Mohamed HAMZA
} 